\title{
Comparison of clinical efficacy of metoprolol combined with irbesartan and hydrochlorothiazide and non-invasive ventilator in the emergency treatment of patients with severe heart failure
}

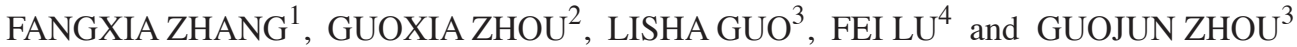 \\ ${ }^{1}$ Department of Cardiology, Binzhou Medical University Hospital, Binzhou, Shandong 256600; \\ ${ }^{2}$ Department of Obstetrics, Jiaozhou Central Hospital of Qingdao, Jiaozhou, Shandong 266300; \\ Departments of ${ }^{3}$ Emergency and ${ }^{4}$ Continuing Education, Binzhou Medical \\ University Hospital, Binzhou, Shandong 256600, P.R. China
}

Received July 31, 2018; Accepted September 26, 2018

DOI: $10.3892 / \mathrm{etm} .2018 .6828$

\begin{abstract}
Clinical efficacy of metoprolol combined with irbesartan and hydrochlorothiazide and non-invasive ventilator in the emergency treatment of patients with severe heart failure (HF) was investigated. A retrospective analysis of the medical records of 124 patients with severe HF admitted to Binzhou Medical University Hospital from May 2012 to August 2016 was performed. Among them, 78 patients who were treated with metoprolol combined with irbesartan and hydrochlorothiazide and non-invasive ventilator for emergency treatment were enrolled into the Research Group, while the Control Group consisted of 46 patients treated with routine medical treatment. Echocardiographic parameters, 6-minute walking test results, the efficacy of the emergency treatment, cardiac function grading, left ventricular ejection fraction (LVEF) and brain natriuretic peptide (BNP) levels were compared between the two groups. After the emergency treatment, the echocardiographic indexes of both groups increased to different extents $(\mathrm{P}<0.05)$. Compared with the results before, patients of both groups walked longer within six minutes after the emergency treatment $(\mathrm{P}<0.05)$. After the emergency treatment, the BNP expression levels in the two groups decreased to different degrees $(\mathrm{P}<0.05)$. After a 7-day emergency treatment, the efficiency rate of treatment of the Research Group was higher than that of the Control Group $(\mathrm{P}<0.05)$. The method is helpful for the recovery of respiratory function, for relieving symptoms in short time, improving
\end{abstract}

Correspondence to: Dr Guojun Zhou, Department of Emergency, Binzhou Medical University Hospital, 661 Huangheer Road, Binzhou, Shandong 256600, P.R. China

E-mail: gv56vu@163.com; mailzhou@foxmail.com

Key words: metoprolol, irbesartan and hydrochlorothiazide, non-invasive ventilator, severe heart failure, emergency treatment cardiac function and promising high safety, using metoprolol and irbesartan and hydrochlorothiazide combined with noninvasive ventilator had satisfactory clinical efficacy in the emergency treatment of patients with severe heart failure and is thus worthy of clinical promotion.

\section{Introduction}

As a clinical comprehensive disease with a change of the cardiac function aroused by various factors, heart failure (HF, mainly refers to the failure of the heart muscle), generally has such clinical symptoms as tachypnea, dyspnea, cyanosis, tachycardia, and frothy sputum (1). Causes of HF are manifold, including myocardial overwork, cardiovascular and cerebrovascular diseases, autoimmune diseases, and sudden decrease of cardiac output caused by immune system diseases, a decreased ventricular pumping function or myocardial contractile function (2). In recent years, the morbidity of $\mathrm{HF}$ is ever-increasing, with elderly people occupying a large proportion of the patients. Characterized by high mortality, high morbidity, and high repeated hospitalization rate (3), HF has become the main killer of elderly patients with chronic diseases (4). Without a timely and effective treatment and with the ever-worsening condition, patients with HF are vulnerable to repeated hospitalization and changed heart structure, thus suffering from severe HF which has higher mortality and is much harder to cure (5). According to statistics, more than 26 million people in the world have been diagnosed with HF, approximately $54.35 \%$ of whom have died within 4 years after diagnosis (6). The common pathogenesis is often related to endocrine disorders, abnormal systemic circulatory function, and myocardial dysfunction (7). Routine emergency treatment of this tricky severe HF using diuretics, invasive oxygen supply, cardiotonic (8) generally brings poor efficacy.

As a $\beta$-blocker, recently metoprolol has been found to be beneficial in relief of HF by relieving the symptoms of the drug users since it can indirectly inhibit the renin-angiotensin system, dilate peripheral blood vessels, significantly reduce 
water-sodium retention (9), reduce myocardial injury aroused by excessive myocardial calcium ion, lower blood pressure through stabling heart rate and reducing cardiac output (10). Irbesartan is an angiotensin-II receptor blocker that reduces the preload and afterload of the heart and effectively dilates the blood vessels (11). Hydrochlorothiazide is a diuretic that activates the sympathetic nervous system and the reninangiotensin system and increases the renin activity in blood generally (12), which when combined with irbesartan that can eliminate diuretic-induced hypokalemia, can better activate renin-angiotensin activity, improve drug safety and reduce adverse reactions (13). Brain natriuretic peptide (BNP), often used as a quantitative marker for HF in clinical practice, is a peptide hormone mainly produced in the ventricle and has active amino acid fragments (14). The occurrence, development, severity of symptoms and prognosis of HF are directly related to BNP. The higher the BNP, the more severe the symptoms. Studies showed that metoprolol combined with irbesartan and hydrochlorothiazide can reduce BNP and improve cardiac functions in HF patients (15).

Mechanical ventilation can be divided into non-invasive ventilation and invasive which are currently very effective in treating dyspnea, a common symptom of HF patients (16). Invasive ventilation refers to the method of assisting breathing through a tracheal intubation or tracheotomy connected to a Continuous Positive Airway Pressure; while noninvasive ventilation (NIV) refers to the use of a Continuous Positive Airway Pressure through a nasal mask to achieve positive pressure ventilation and increase alveolar ventilation without tracheal intubation (17). After repeated research worldwide, the safety, efficacy and economic practicability of NIV have been confirmed because it reduce the possibility of concurrent infection and adverse reactions caused by invasive mechanical ventilation, and improve long-term prognosis (18). Continuous Positive Airway Pressure is commonly used in the treatment of patients with sleep apnea syndrome and pulmonary respiratory failure, and shows good results. With the advancement of science and technology, the continuously improved NIV technology has gradually gained much attention from cardiologists as non-invasive ventilator can effectively reduce the heart preload, correct hypoxemia and acidosis, promote the efficacy of anti-heart failure drugs, and prevent cardiac-respiratory arrest caused by hypoxia $(19,20)$.

There is an urgent need to find a safer and more effective severe HF emergency treatment plan, considering the yearly-increasing incident cases and the unsatisfactory current treatment. To this end, this study explored the clinical efficacy of metoprolol combined with irbesartan and hydrochlorothiazide and non-invasive ventilator in the emergency treatment of patients with severe $\mathrm{HF}$, looking for a safe and effective severe HF emergency treatment plan, providing a further scientific basis for the clinical use.

\section{Patients and methods}

Clinical baseline data. This study retrospectively analyzed the medical records of 124 patients with severe HF admitted to the Emergency Department of Binzhou Medical University Hospital (Binzhou, China) from May 2012 to August 2016 and who all met the diagnostic criteria for heart failure. Among them,
78 patients treated with metoprolol combined with irbesartan and hydrochlorothiazide and non-invasive ventilator for emergency treatment were enrolled into the Research Group, including 41 male patients and 37 female patients with an average age of $71.34 \pm 5.26$, while the Control Group, consisted of 46 patients ( 26 men and 20 women) treated with routine medical treatment in the emergency depart, with an average age of $70.41 \pm 6.39$. The study was conducted after approval by the Medical Ethics Committee of Binzhou Medical University Hospital. Written informed consent was obtained from patients and their families.

Criteria for inclusion and exclusion. Inclusion criteria: age $>60$ years; left ventricular ejection fractions (LVEF) $\leq 0.35$; enlargement of left ventricular showed by echocardiography; graded NYHA cardiac function as Level 4 and above; intact structure of upper respiratory tract without excessive respiratory secretion; stable hemodynamics.

Exclusion criteria: those who were allergic to drugs used in this study such as metoprolol, irbesartan or hydrochlorothiazide; those with severe angina pectoris, myocardial infarction or liver and kidney dysfunction; those with systolic blood pressure $<90 \mathrm{mmHg}$; those with malignant tumors; those uncooperative with the treatment; those with serious infections.

Emergency treatment plan for the Research Group. Patients in the Research Group adopted a low-sodium diet, strictly controlled amount of drinking water, and enough rest during the treatment. Patients were treated with metoprolol (manufacturer: AstraZeneca plc., code number of medical product approved by SFDA: medical product approved by SFDA H32025391) combined with irbesartan and hydrochlorothiazide (manufacturer: Zhejiang Huahai Pharmaceutical Co., Ltd., Zhejiang, China; code number of medical product approved by SFDA: medical product approved by SFDA H20058709) and non-invasive ventilator (manufacturer: Respironics Inc; product model: BiPAP-Vision) for emergency treatment. Patients took orally metoprolol $25 \mathrm{mg} /$ day, twice a day, irbesartan and hydrochlorothiazide (150 mg for irbesartan/ and $12.50 \mathrm{mg}$ for hydrochlorothiazide)/day, twice a day. The Research Group used the non-invasive ventilator with the S/T mode and automatic parameter setting. Oxygen was directly connected to the mask which was in close contact with the patient's face to avoid oxygen leakage. The inspiratory positive airway pressure and the expiratory positive airway pressure were set separately with an initial auxiliary ratio of $85 \%$. According to the relief of the patient's breathing difficulties, the auxiliary ratio was gradually reduced to 70,50 , and $30 \%$. The positive airway pressure of the expiratory term was set to 5-6 $\mathrm{cm} \mathrm{H}_{2} \mathrm{O}$; the positive airway pressure of the inspiratory item was set to $6 \mathrm{~cm} \mathrm{H}_{2} \mathrm{O}$, and the initial oxygen concentration was $100 \%$. The NIV time was extended as much as possible until the patient's breathing difficulties were significantly relieved, then the ventilation time was gradually shortened, and the ventilation pressure was reduced until offline. One week was a course of treatment.

Emergency treatment plan for the Control Group. The Control Group was guaranteed a low-sodium diet, strictly controlled amount of drinking water, and enough rest during 
Table I. Grading criteria of cardiac function.

Levels

Degree of activity of HF patients

Level 1

The patients have heart disease, but the amount of daily activities is not limited.

General physical activities do not cause excessive fatigue, palpitations, asthma or angina.

Level 2 Physical activities in patients with heart disease are slightly limited. No self-consciousness at rest. General physical activities can cause excessive fatigue, palpitations, asthma or angina.

Level 3 The patients have heart disease, so physical activities are significantly limited. Asymptomatic at rest, but activities with lower labor intensity than general physical activities can cause excessive fatigue, palpitations, asthma or angina.

Level 4 Patients with heart disease can not engage in any physical activity, and symptoms of heart failure occur at rest, which is aggravated after physical activities.

treatment. Patients were prescribed to take orally furosemide tablets (manufacturer: Dongbei Pharmaceutical Group Company Shenyang No. 1 Pharmaceutical Co., Ltd., Shenyang, China; code number of medical product approved by SFDA: medical product approved by SFDA H21022890) $40 \mathrm{mg} /$ day, twice a day; to take nitroglycerin through sublingual administration (manufacturer: Beijing Yimin Pharmaceutical Plant, Beijing, China; code number of medical product approved by SFDA: medical product approved by SFDA H11021022) 0.25-0.5 mg/time (can be re-administered once after $5 \mathrm{~min}$ ), 1-2 times a day; to take intravenous infusion of sodium nitroprusside (light avoided) (manufacturer: Henan Wanxi Pharmaceutical Co., Ltd., Henan, China; code number of medical product approved by SFDA: medical product approved by SFDA H20143166), which was diluted with $5 \%$ glucose solution (each kilogram of the patients' weight required $30 \mu \mathrm{g} /$ min, 12 h/day). One week was a course of treatment.

\section{Detection indicators}

Echocardiography. After admitted to the emergency department of internal medicine, the patients were examined by Color Doppler Echocardiography (manufacturer: Hewlett-Packard Co., Palo Alto, CA, USA, model: HP77020Ac). All patients were treated with the same Doppler ultrasound system and the same doctor performed the examination. Cardiac index (CI), stroke volume (SV), cardiac output (CO), and fractional shortening (FS) were recorded before and after treatment.

$L V E F$. LVEF is the percentage of stroke volume to the ventricular end-diastolic volume. The volume of ventricular diastolic is approximately $145 \mathrm{ml}$ in the left ventricle, approximately $138 \mathrm{ml}$ in the right ventricle, and the SV is approximately 60-80 ml. Cardiac color Doppler examination is an important indicator for diagnosing HF. Under normal circumstances, $\mathrm{LVEF} \geq 0.5$, right ventricular ejection fraction $\geq 0.4$, the results less than normal scores can prove cardiac dysfunction.

Six-minute walk experiment. The experiment was a test for the motor function status of patients with moderate to severe HF (21), which examined the motion state of patients from both the Research and Control Groups before the treatment, 1 day after the treatment, and 7 days after the treatment. The test site was an unimpeded corridor with one chair placed every 15 meters for the patients to rest halfway. The patients were told to walk as far as possible within the prescribed site to record the longest distance the patients walked within 6 min. During the walking, verbal encouragements such as 'well done, please go on' were delivered every fixed time. Break during the walk was allowed, but moving on after physical recovery was strongly required.

Examination of BNP. BNP content in the serum is an important indicator for assessing cardiac function. The more severe the $\mathrm{HF}$ is, the higher the BNP expression level in the serum is. Blood samples taken from the elbow venous of all patients before the treatment, 1 day after the treatment, and 7 days after the treatment were sent to the professional inspectors in the laboratory of the hospital. The test method was enzyme-linked immunosorbent assay, the kits were from Hangzhou Zheda Dixun Biological Gene Engineering Co., Ltd. (Hangzhou, China) with a product code of 59400364827

Judging criteria of the efficacy of emergency treatment. Effective: After the emergency treatment, the symptoms of HF patients were relieved. The BNP expression level decreased, and the LVEF score increased. Ineffective: After the emergency treatment, the symptoms of HF patients were not relieved or even aggravated, and the cardiac function was unchanged or worsened.

Grading criteria of cardiac function. According to the degree of activity of the HF patients, the impairment of cardiac function was divided into 4 levels, as shown in Table I.

\section{Results}

Comparison between the clinical baseline data of two groups. According to the comparison between the two groups in terms of age, sex, body mass index (BMI), LVEF, blood glucose, heart rate, systolic blood pressure, diastolic blood pressure, mean arterial pressure, CI, SV, FS and CO, no statistical difference was shown between the two groups $(\mathrm{P}>0.05)$ (Table II).

Changes of echocardiography test results after the emergency treatment. After 7 days of the emergency treatment, the indexes of echocardiography of the Research and Control Groups were increased to different degrees. Statistical difference was indicated between the two groups in terms of LVEF as the LVEF 
Table II. Comparison of the clinical baseline data between the Research Group and Control Group [n (\%)] (mean \pm SD).

\begin{tabular}{|c|c|c|c|c|}
\hline Terms & The Research Group $(n=78)$ & The Control Group $(\mathrm{n}=46)$ & $\chi^{2} / \mathrm{t}$ value & P-value \\
\hline Age (year) & & & 0.253 & 0.708 \\
\hline$\leq 70$ & 32 & 21 & & \\
\hline$>70$ & 46 & 25 & & \\
\hline Sex & & & 0.182 & 0.712 \\
\hline Male & 41 & 26 & & \\
\hline Female & 37 & 20 & & \\
\hline $\operatorname{BMI}\left(\mathrm{kg} / \mathrm{m}^{2}\right)$ & & & 0.308 & 0.683 \\
\hline$<24$ & 24 & 14 & & \\
\hline$\geq 24$ & 54 & 32 & & \\
\hline LVEF & & & 0.976 & 0.424 \\
\hline$\leq 0.30$ & 51 & 34 & & \\
\hline$>0.30$ & 27 & 12 & & \\
\hline Blood glucose (mmol/l) & & & 0.150 & 0.712 \\
\hline$\leq 8$ & 35 & 19 & & \\
\hline$>8$ & 43 & 27 & & \\
\hline Heart rate (bpm) & $123.13 \pm 14.24$ & $125.34 \pm 12.24$ & 0.878 & 0.382 \\
\hline Systolic blood pressure (mmHg) & $156.34 \pm 12.34$ & $157.43 \pm 13.21$ & 0.463 & 0.644 \\
\hline Diastolic blood pressure $(\mathrm{mmHg})$ & $88.32 \pm 4.25$ & $86.68 \pm 6.31$ & 1.727 & 0.087 \\
\hline Mean arterial pressure $(\mathrm{mmHg})$ & $132.32 \pm 7.43$ & $134.53 \pm 7.43$ & 1.600 & 0.112 \\
\hline $\mathrm{CI}\left(1 / \mathrm{min} / \mathrm{m}^{2}\right)$ & $1.85 \pm 0.44$ & $1.92 \pm 0.31$ & 1.084 & 0.281 \\
\hline SV (ml) & $37.31 \pm 5.33$ & $35.78 \pm 6.88$ & 1.383 & 0.169 \\
\hline FS $(\%)$ & $16.43 \pm 6.29$ & $17.11 \pm 5.32$ & 0.615 & 0.540 \\
\hline $\mathrm{CO}(1 / \mathrm{min})$ & $1.35 \pm 0.28$ & $1.40 \pm 0.23$ & 1.024 & 0.308 \\
\hline
\end{tabular}

Table III. The efficacy of emergency treatment and comparison of cardiac function grading between the two groups after the emergency treatment.

\begin{tabular}{|c|c|c|c|c|}
\hline Terms & The Research Group (n=78) & The Control Group $(n=46)$ & $\chi^{2}$ value & P-value \\
\hline Efficacy & & & 6.426 & 0.016 \\
\hline Effective & $74(94.87)$ & $37(80.43)$ & & \\
\hline Ineffective & $4(5.13)$ & 9 (19.57) & & \\
\hline \multicolumn{5}{|c|}{ Cardiac function grades } \\
\hline 1 & $2(2.56)$ & $0(0.00)$ & 1.199 & 0.530 \\
\hline 2 & $23(29.49)$ & $6(13.04)$ & 4.367 & 0.048 \\
\hline 3 & $42(53.85)$ & $16(34.78)$ & 4.224 & 0.043 \\
\hline 4 & $11(14.10)$ & $24(52.17)$ & 20.702 & $<0.001$ \\
\hline
\end{tabular}

of the Research Group was $0.31 \pm 0.07$ on the 1 st and $0.38 \pm 0.05$ on the 7 th day after the emergency treatment, higher than that of the Control Group $(0.28 \pm 0.06)$ on the 1 st and 7 th day $(0.32 \pm 0.06)$ after the emergency treatment $(\mathrm{P}<0.05)$. Statistical difference existed between the two groups since the CI results of the Research Group on the 1st and 7th day after treatment were $2.86 \pm 0.42 \mathrm{l} / \mathrm{min} / \mathrm{m}^{2}, 3.83 \pm 0.54 \mathrm{l} / \mathrm{min} / \mathrm{m}^{2}$ respectively, which were higher than those of the Control Group on the 1st $\left(2.31 \pm 0.36 \mathrm{l} / \mathrm{min} / \mathrm{m}^{2}\right)$ and 7 th day $\left(2.79 \pm 0.421 / \mathrm{min} / \mathrm{m}^{2}\right)$ after the emergency treatment $(\mathrm{P}<0.05)$. The two groups proved to be statistically different by the results that the SV of the Research Group 1 and 7 days after the emergency treatment was $48.65 \pm 7.43 \mathrm{ml}$, and $63.43 \pm 5.67 \mathrm{ml}$, respectively, higher than $43.35 \pm 6.95 \mathrm{ml}$ on the 1 st day and $52.35 \pm 5.42 \mathrm{ml}$ on the 7 th day after treatment of the Control Group $(\mathrm{P}<0.05)$. The FS of the Research Group 1 and 7 days after the emergency treatment was $20.21 \pm 5.32 \%$, and $24.45 \pm 4.79 \%$, respectively, higher than $18.11 \pm 5.67 \%$ on the 1 st day and $20.30 \pm 4.93 \%$ on the 7 th day after treatment of the Control Group $(\mathrm{P}<0.05)$. The CO of the Research Group 1 and 7 days after the emergency treatment 

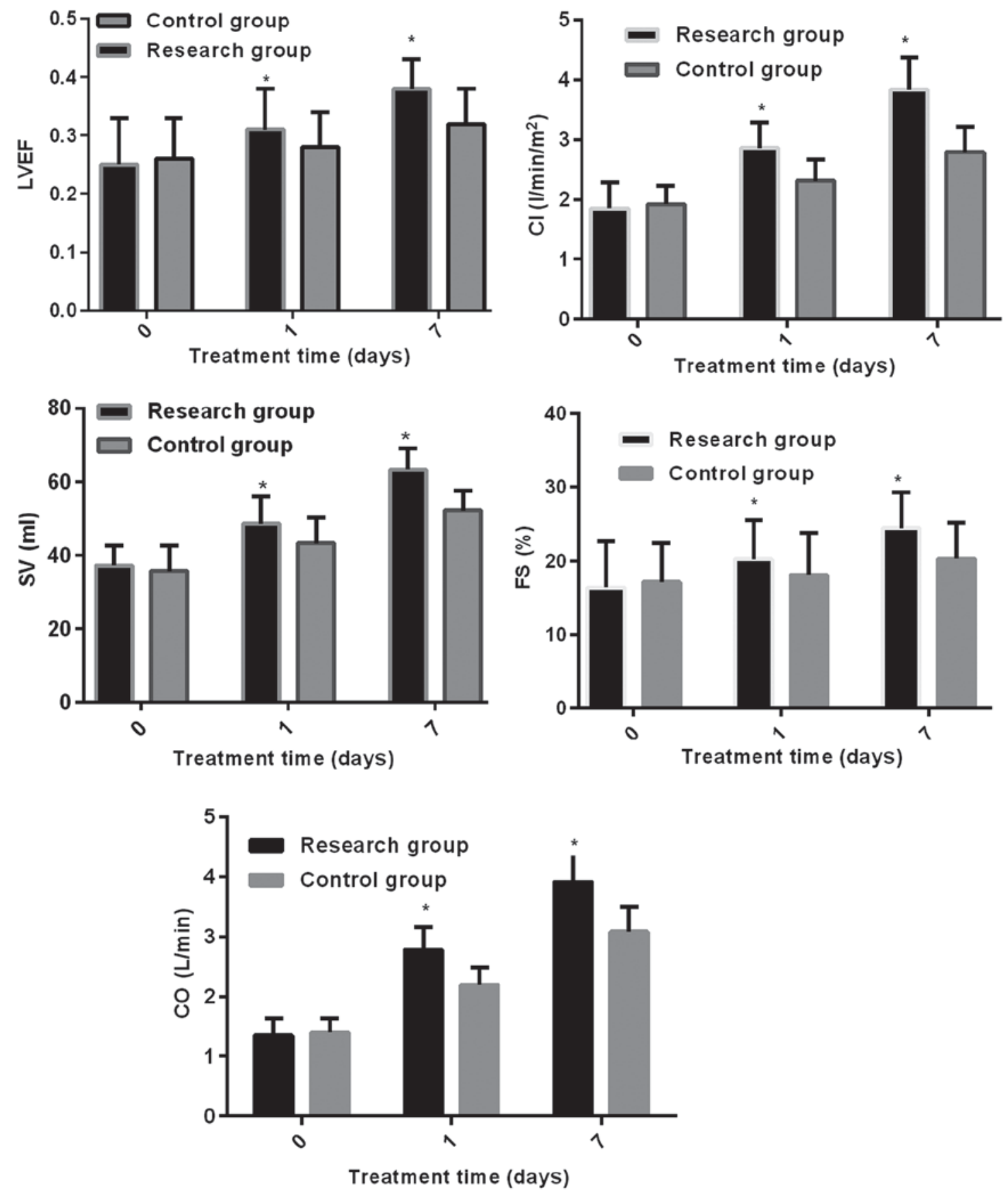

Figure 1. No significant differences in the LVEF, CI, SV, FS, and CO indexes between the Research and Control Groups before the emergency treatment $(\mathrm{P}>0.05)$. After the emergency treatment, all indexes of the echocardiogram of both groups were increased, with indexes of the Research Group being higher than those of the Control Group $(\mathrm{P}<0.05)$. The Research Group was significantly different from the Control Group ( $\left.{ }^{*} \mathrm{P}<0.05\right)$.

was $2.78 \pm 0.37 \mathrm{l} / \mathrm{min}$, and $3.91 \pm 0.47 \mathrm{l} / \mathrm{min}$, respectively, higher than $2.19 \pm 0.29 \mathrm{l} / \mathrm{min}$ on the 1 st day and $3.08 \pm 0.42 \mathrm{l} / \mathrm{min}$ on the 7th day after treatment of the Control Group $(\mathrm{P}<0.05)$ (Fig. 1).

Comparison of the 6-minute walking test of the two groups before and after the emergency treatment. No statistical difference in terms of the 6-minute walk test before emergency treatment between the two groups was seen since the test result of the Study Group was $343.35 \pm 52.57$ meters and the Regular Group was $342.88 \pm 54.43$ meters before the emergency treatment $(t=0.047, P=0.962)$, while after the seven-day long emergency treatment, the length of walking of the Study Group 1 day after the emergency treatment was
$388.45 \pm 65.39$ meters, longer than the $361.45 \pm 62.91$ meters of the Regular Group $(\mathrm{t}=2.252, \mathrm{P}=0.026)$. The length of walking of the Study Group 7 days after the emergency treatment was $417.56 \pm 48.94$ meters, longer than the $386.71 \pm 55.83$ meters of the Regular Group ( $\mathrm{t}=3.217$, $\mathrm{P}=0.002)$ (Fig. 2).

Changes of the serum BNP expression level of the two groups before and after the emergency treatment. No statistical difference in terms of the BNP expression level before the emergency treatment between the two groups was detected since the BNP expression of the Study Group was $1096.43 \pm 103.53$, similar to $1091.54 \pm 112.42$ of the Regular Group $(\mathrm{t}=0.246, \mathrm{P}=0.806)$. 


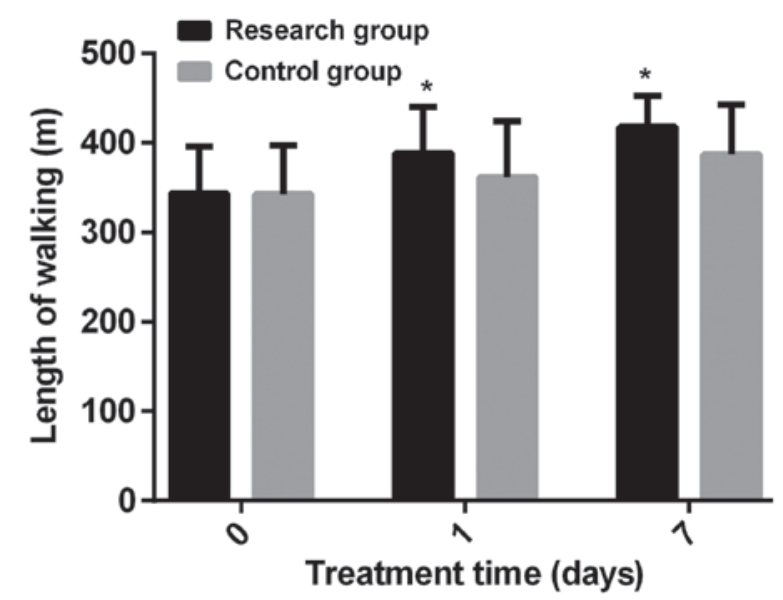

Figure 2. No significant difference was found in the walking length within 6 min between the Research and Control Groups before the emergency treatment $(\mathrm{t}=0.047, \mathrm{P}=0.962)$. After the emergency treatment, the length of the walk in $6 \mathrm{~min}$ in the two groups increased to varying degrees, with the walking length of the Research Group being longer than that of the Control Group $(\mathrm{P}<0.05)$. The Research Group was significantly different from the Control Group $\left({ }^{*} \mathrm{P}<0.05\right)$.

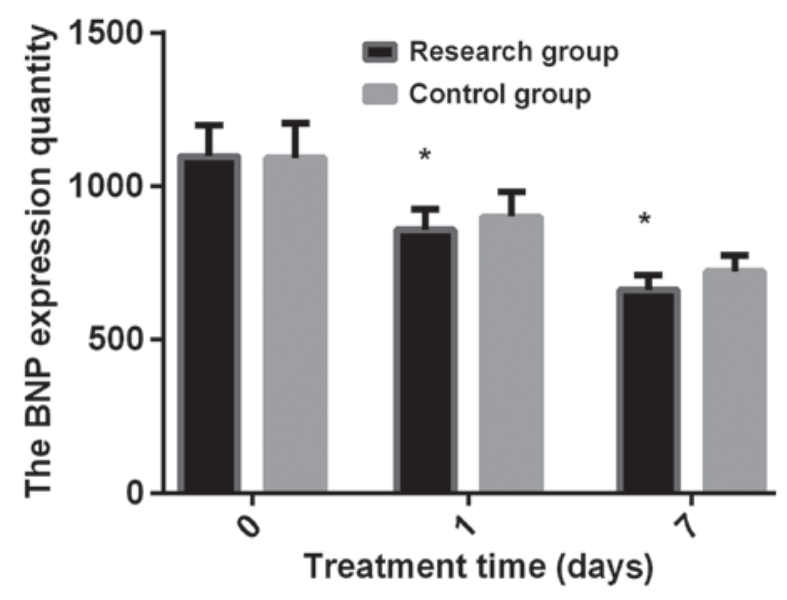

Figure 3. Enzyme-linked immunosorbent assay showed, no significant differences in the expression of BNP in the serum between the Research and Control Groups before the emergency treatment $(\mathrm{t}=0.246, \mathrm{P}=0.806)$. After the emergency treatment, the serum BNP levels of both groups decreased to different degrees, with the serum BNP level of the Research Group being lower than that of the Control Group $(\mathrm{P}<0.05)$. The Research Group was significantly different from the Control Group $\left({ }^{*} \mathrm{P}<0.05\right)$.

After seven days of the emergency treatment, the BNP expression of the Research Group was $857.53 \pm 67.611$ day after the emergency treatment, lower than the $900.53 \pm 79.82$ of the Control Group ( $\mathrm{t}=3.197, \mathrm{P}=0.018)$. The $\mathrm{BNP}$ expression of the Research Group was 662.34 $446.78,7$ days after the emergency treatment, lower than the $721.55 \pm 52.83$ of the Control Group $(\mathrm{t}=6.487, \mathrm{P}<0.001)($ Fig. 3).

The efficacy of emergency treatment and comparison of cardiac function grading between the two groups after the emergency treatment. After a 7-day emergency treatment, the efficiency rate of treatment of the Research Group was $94.87 \%$, higher than that of the Control Group $(80.43 \%)$ $\left(\chi^{2}=6.426, \mathrm{P}=0.016\right)$, which explained why 2 patients from the Research Group and no patient from the Control Group were graded as Level 1 in terms of the heart function (no difference between the two groups, $\chi^{2}=1.199, \mathrm{P}=0.530$ ). In addition, 23 patients from the Research Group and 6 patients from the Control Group were graded as Level $2\left(\chi^{2}=4.367, \mathrm{P}=0.048\right)$, while 42 patients from the Research Group and 16 patients from the Control Group were graded as Level $3(\mathrm{P}<0.05$, $\left.\chi^{2}=4.367, \mathrm{P}=0.048\right)$. Finally, 11 patients from the Research Group and 24 patients from the Control Group were graded as Level $4\left(\chi^{2}=20.702, \mathrm{P}<0.001\right)$ (Table III).

\section{Discussion}

HF is a common internal medical disease manifesting as ventricular filling and impaired ejection ability with the complication of obstructive pulmonary disease, caused by a variety of factors, such as autoimmune diseases, tumors, cardiogenic diseases and metabolic diseases (22). HF has a gradually increasing mortality as the patients get older, becoming an important reason for the abnormal death of the elderly in the contemporary era. The disease of HF has sudden and severe symptoms and rapid development to destroy the cardiac function to produce enough cardiac output to meet the daily metabolic needs of the body, and easily turn into severe HF without timely treatment (23), which has extremely high mortality and poor prognosis, leading to an average survival time of less than 5 years (24). Emergency treatment is an important part of severe HF rescue treatment, so active and effective emergency treatment is the key to improve the prognosis, improve the survival rate of patients with severe HF, and improve the patients' quality of life (25).

Studies have pointed out that when patients suffered from heart failure, noradrenergic nerves in the body would be over-activated, causing necrosis and apoptosis of cardiomyocytes directly through cytotoxicity, promoting hypertrophy of cardiac fibroblasts, leading to myocardial fibrosis and abnormal hypertrophy (26). $\beta$-blockers can effectively block the excessive activation of the noradrenergic nervous system, improve cardiac function in patients with severe $\mathrm{HF}$, and block ventricular remodeling (27). In patients with severe HF, pulmonary compliance is weakened, resulting in increased airway resistance and dysfunction in ventilation. Clinically, sudden dyspnea and cyanosis are common for patients with severe HF, and can be life-threatening in severe cases. The method of mechanical ventilation is often used in the clinical emergency treatment of severe HF patients as it can help patients breath, increase inspiratory volume, increase myocardial oxygen supply, reduce cardiac burden, improve oxygenation (28). Compared with traditional invasive mechanical ventilation, NIV has advantages of no need to open the trachea of severe HF patients, no need for intubation, eliminating the corresponding serious complications, decreasing tracheal loss, and reducing medical expenses (29). The study made by Kuhn et al (30) found that NIV achieved good results in the emergency treatment of patients with acute left heart failure and contributed to the recovery of patients. Irbesartan is a powerful angiotensin-II receptor blocker, and hydrochlorothiazide a common diuretic, and the combination of the two is a common compound preparation for the treatment of essential hypertension, beneficial to activating sympathetic nerve and renin-angiotensin, inhibiting the decline of serum 
potassium level, improving medication safety, and reducing adverse reactions (31). A study (32) showed that irbesartan and hydrochlorothiazide combined with conventional medication for severe HF could effectively reduce the adverse reactions and recurrence rate, and promise high safety. As commonly used drugs in severe HF, irbesartan and hydrochlorothiazide do not show good clinical efficacy to improve the symptoms of patients. Chrysant et al (33) found that irbesartan and hydrochlorothiazide combined with metoprolol in the treatment of chronic elderly patients with HF could significantly improve the efficacy and cardiac function, which agrees with our study results that metoprolol combined with and irbesartan and hydrochlorothiazide and non-invasive ventilator had a good effect in the emergency treatment of patients with severe HF, conducive to the recovery of cardiac function, the relief of the patient's breathing difficulties and the reduction of the preload and afterload of the heart.

The results of the experiment showed no obvious difference between the echocardiographic indexes of the Research Group and the Control Group ( $>>0.05)$, but after the emergency treatment, the echocardiographic indexes of both groups were increased to different degrees, with the LVEF, CI, SV, FS, and CO of the echocardiogram of the Research Group on the 1st and 7th day after the emergency treatment being higher than that of the Control Group $(\mathrm{P}<0.05)$. The results of the experiment indicated that the emergency treatment using metoprolol and irbesartan and hydrochlorothiazide combined with non-invasive ventilator helped to recover normal cardiac function, to increase cardiac output, to normalize the ventricular ejection of severe HF patients. Before the emergency treatment, the Research Group and the Control Group walked similar length within 6 min $(\mathrm{P}>0.05)$. After the emergency treatment, the length of walking in the two groups was extended, with the walking length of the Research Group on the 1st and 7th day after emergency treatment being longer than that of the Control Group $(\mathrm{P}<0.05)$, which showed that the use of metoprolol and irbesartan and hydrochlorothiazide combined with non-invasive ventilator for emergency treatment of severe HF patients could quickly restore normal body function and improve clinical symptoms such as dyspnea in patients with severe HF. The patient has clinical symptoms such as difficulty in breathing. No difference in serum BNP expression was detected between the Research and Control Groups before the emergency treatment $(\mathrm{P}>0.05)$. After the emergency treatment, the BNP expression levels in the two groups decreased in different degrees, with the BNP expression level of the Research Group on the 1st and 7 th day after the emergency treatment was lower than that of the Control Group $(\mathrm{P}<0.05)$, showing that the use of metoprolol and irbesartan and hydrochlorothiazide combined with non-invasive ventilator for the emergency treatment of severe HF patients could relieve the symptoms in patients, and bring better prognosis if the severity of disease development was lower. After the 7-day emergency treatment, the efficiency rate of treatment of the Research Group was $94.87 \%$, higher than that of the Control Group (80.43\%) $(\mathrm{P}<0.05)$, which explained why 2 patients from the Research Group, but no patient from the Control Group was graded as Level 1 in terms of the heart function. In addition, 23 patients from the Research Group and 6 patients from the Control Group were graded as Level $2(\mathrm{P}<0.05)$, while 42 patients from the Research Group and 16 patients from the Control Group were graded as Level $3(\mathrm{P}<0.05)$. At last, 11 patients from the Research Group and 24 patients from the Control Group were graded as Level $4(\mathrm{P}<0.05)$. The results of this study indicated that the method of using metoprolol and irbesartan and hydrochlorothiazide combined with non-invasive ventilator for the emergency treatment of patients with severe HF was more efficient.

However, a limitation of this study was that the method of retrospective analysis did not achieve perfection in the collection of data of clinical and special examinations for patients, and the limited number of cases admitted was not capable of offering sufficient data for statistical comparison.

In summary, the method of using metoprolol and irbesartan and hydrochlorothiazide combined with non-invasive ventilator in the emergency treatment of severe HF patients proved to be of high safety and is worth clinical promotion because it was significantly efficient to help patients with the recovery of respiratory function, to relieve symptoms in short time and improve cardiac function.

\section{Acknowledgements}

Not applicable.

\section{Funding}

No funding was received.

\section{Availability of data and materials}

The datasets used and/or analyzed during the present study are available from the corresponding author on reasonable request.

\section{Authors' contributions}

FZ, GZ, and LG conceived and designed the study, compared the 6-minute walking test of the two groups before and after the emergency treatment and analyzed the echocardiography result. FL and GZ examined the serum BNP expression level of the two groups before and after the emergency treatment. All authors have read and approved the final manuscript.

\section{Ethics approval and consent to participate}

The study was approved by the Ethics Committee of Binzhou Medical University Hospital (Binzhou, China). Patients who participated in this research had complete clinical data. Signed written informed consents were obtained from the patients and/or guardians.

\section{Patient consent for publication}

Not applicable.

\section{Competing interests}

The authors declare that they have no competing interests. 


\section{References}

1. Chang AM, Maisel AS and Hollander JE: Diagnosis of heart failure. Heart Fail Clin 5: 25-35, 2009.

2. Li JD, Cheng AY, Huo YL, Fan J, Zhang YP, Fang ZQ, Sun HS, Peng W, Zhang JS, Wang HP, et al: Bilateral renal denervation ameliorates isoproterenol-induced heart failure through downregulation of the brain renin-angiotensin system and inflammation in rat. Oxid Med Cell Longev 2016: 3562634, 2016.

3. Díez J: Serelaxin: A novel therapy for acute heart failure with a range of hemodynamic and non-hemodynamic actions. Am J Cardiovasc Drugs 14: 275-285, 2014.

4. Valbusa F, Agnoletti D, Scala L, Grillo C, Arduini P, Bonapace S, Calabria S, Scaturro G, Mantovani A, Zoppini G, et al: Non-alcoholic fatty liver disease and increased risk of all-cause mortality in elderly patients admitted for acute heart failure. Int J Cardiol 265: 162-168, 2018.

5. Davarzani N, Sanders-van Wijk S, Karel J, Maeder MT, Leibundgut G, Gutmann M, Pfisterer ME, Rickenbacher P, Peeters R and Brunner-la Rocca HP: N-terminal pro-B-type natriuretic peptide-guided therapy in chronic heart failure reduces repeated hospitalizations-results from TIME-CHF. J Card Fail 23: 382-389, 2017.

6. Böhm M, Voors AA, Ketelslegers JM, Schirmer SH, Turgonyi E, Bramlage P and Zannad F: Biomarkers: Optimizing treatment guidance in heart failure. Clin Res Cardiol 100: 973-981, 2011.

7. Aikawa T, Naya M, Obara M, Manabe O, Tomiyama Y, Magota K, Yamada S, Katoh C, Tamaki N and Tsutsui H: Impaired myocardial sympathetic innervation is associated with diastolic dysfunction in heart failure with preserved ejection fraction: 11C-Hydroxyephedrine PET Study. J Nucl Med 58: 784-790, 2017.

8. Qavi AH, Kamal R and Schrier RW: Clinical use of diuretics in heart failure, cirrhosis, and nephrotic syndrome. Int $\mathbf{J}$ Nephrol 2015: 975934, 2015.

9. Mao Y, Tokudome T and Kishimoto I: Ghrelin as a treatment for cardiovascular diseases. Hypertension 64: 450-454, 2014.

10. Kotecha D, Holmes J, Krum H, Altman DG, Manzano L, Cleland JG, Lip GY, Coats AJ, Andersson B, Kirchhof P, et al; Beta-Blockers in Heart Failure Collaborative Group: Efficacy of $\beta$ blockers in patients with heart failure plus atrial fibrillation: An individual-patient data meta-analysis. Lancet 384: 2235-2243, 2014.

11. Massie BM, Carson PE, McMurray JJ, Komajda M, McKelvie R, Zile MR, Anderson S, Donovan M, Iverson E, Staiger C, et al; I-PRESERVE Investigators: Irbesartan in patients with heart failure and preserved ejection fraction. N Engl J Med 359: 2456-2467, 2008.

12. Luo J, Gao X, Peng L, Sun H and Dai G: Effects of hydrochlorothiazide on cardiac remodeling in a rat model of myocardial infarction-induced congestive heart failure. Eur J Pharmacol 667: 314-321, 2011.

13. Zhang JL, Yu H, Hou YW, Wang K, Bi WS, Zhang L, Wang Q, $\mathrm{Li}$ P, Yu ML and Zhao XX: Impact of long-term potassium supplementation on thiazide diuretic-induced abnormalities of glucose and uric acid metabolisms. J Hum Hypertens 32: 301-310, 2018.

14. Mowla MM and Bustami BB: Brain natriuretic peptide in heart failure and beyond. Saudi Med J 27: 1457-1461, 2006.

15. Ghali JK, Piña IL, Gottlieb SS, Deedwania PC and Wikstrand JC; MERIT-HF Study Group: Metoprolol CR/XL in female patients with heart failure: Analysis of the experience in Metoprolol Extended-Release Randomized Intervention Trial in Heart Failure (MERIT-HF). Circulation 105: 1585-1591, 2002.

16. Esquinas AM and Kasai T: Non-invasive mechanical ventilation for sleep disordered breathing and cardiac function in chronic heart failure. More CPAP or more ASV? That is the question. Int J Cardiol 168: 2978, 2013.

17. Gregoretti C, Mattei A and Carlucci A: Noninvasive ventilation outside the critical care unit. In: Practical Issues Updates in Anesthesia and Intensive Care. Springer International Publishing, 139-147, 2015.
18. Dave C, Turner A, Dretzke J, Bayliss S, O'Brien D, Jowett S and Moore D: Protocol for a systematic review and economic evaluation of the clinical and cost-effectiveness of non-hospitalbased non-invasive ventilation (NIV) in patients with stable end-stage COPD with hypercapnic respiratory failure. Syst Rev 3: 32, 2014.

19. Panacek EA and Kirk JD: Role of noninvasive ventilation in the management of acutely decompensated heart failure. Rev Cardiovasc Med 3 (Suppl 4): S35-S40, 2002.

20. Stoltzfus S: The role of noninvasive ventilation: CPAP and BiPAP in the treatment of congestive heart failure. Dimens Crit Care Nurs 25: 66-70, 2006.

21. Prescher S, Schoebel C, Koehler K, Deckwart O, Wellge B, Honold M, Hartmann O, Winkler S and Koehler F: Prognostic value of serial six-minute walk tests using tele-accelerometry in patients with chronic heart failure: A pre-specified sub-study of the TIM-HF-Trial. Eur J Prev Cardiol 23 (Suppl): 21-26, 2016.

22. Barth AS, Kumordzie A, Frangakis C, Margulies KB, Cappola TP and Tomaselli GF: Reciprocal transcriptional regulation of metabolic and signaling pathways correlates with disease severity in heart failure. Circ Cardiovasc Genet 4: 475-483, 2011.

23. No authors listed: 5 action steps for early heart failure. Early heart failure may be mild but can worsen quickly. Learn what you can do to keep symptoms in check. Harv Heart Lett 24: 3 , 2014.

24. Pourafkari L, Ghaffari S, Afshar AH, Anwar S and Nader ND: Predicting outcome in acute heart failure, does it matter? Acta Cardiol 70: 653-663, 2015.

25. Bonacchi M, Vannini A, Harmelin G, Batacchi S, Bugetti M, Sani G and Peris A: Inverted-Takotsubo cardiomyopathy: Severe refractory heart failure in poly-trauma patients saved by emergency extracorporeal life support. Interact Cardiovasc Thorac Surg 20: 365-371, 2015.

26. Patel KP: Role of paraventricular nucleus in mediating sympathetic outflow in heart failure. Heart Fail Rev 5: 73-86, 2000.

27 . Berg T: $\beta 1$-Blockers lower norepinephrine release by inhibiting presynaptic, facilitating $\beta 1$-adrenoceptors in normotensive and hypertensive rats. Front Neurol 5: 51, 2014.

28. Chen Y, Chen P, Hanaoka M, Huang X, Droma Y and Kubo K: Mechanical ventilation in patients with hypoxemia due to refractory heart failure. Intern Med 47: 367-373, 2008.

29. Squadrone E, Frigerio P, Fogliati C, Gregoretti C, Conti G, Antonelli M, Costa R, Baiardi P and Navalesi P: Noninvasive vs invasive ventilation in COPD patients with severe acute respiratory failure deemed to require ventilatory assistance. Intensive Care Med 30: 1303-1310, 2004.

30. Kuhn BT, Bradley LA, Dempsey TM, Puro AC and Adams JY: Management of mechanical ventilation in decompensated heart failure. J Cardiovasc Dev Dis 3: 3, 2016.

31. Huang QF, Sheng CS, Li Y, Ma GS, Dai QY and Wang JG; INCENT Investigators: Efficacy and safety of a fixed combination of irbesartan/hydrochlorothiazide in Chinese patients with moderate to severe hypertension. Drugs R D 13: 109-117, 2013.

32. Croxtall JD and Keating GM: Irbesartan/Hydrochlorothiazide: In moderate to severe hypertension. Drugs 68: 1465-1472, 2008.

33. Chrysant SG, Neutel JM and Ferdinand KC; INCLUSIVE investigators: Irbesartan/hydrochlorothiazide for the treatment of isolated systolic hypertension: A subgroup analysis of the INCLUSIVE trial. J Natl Med Assoc 101: 300-307, 2009.

This work is licensed under a Creative Commons Attribution-NonCommercial-NoDerivatives 4.0 International (CC BY-NC-ND 4.0) License. 